\title{
UTILIZAÇÃO DA ANÁLISE MULTICRITÉRIO PARA DIVISÃO DE RECURSOS FINANCEIROS EM UNIVERSIDADES PÚBLICAS
}

\author{
http://dx.doi.org/10.5902/2318133848510
}

\author{
Kleber Fernandes de Oliveira ${ }^{1}$ \\ Thais Ettinger Oliveira Salgado \\ Marcos Eduardo Zambanini ${ }^{3}$ \\ Jussara Maria Moreno Jacintho ${ }^{4}$
}

\begin{abstract}
Resumo
Neste artigo propõe-se um modelo baseado na análise multicritério de apoio à tomada de decisão - Prometheé - para auxiliar na descentralização de recursos de custeio em universidades públicas. Utilizou-se de dados secundários e indicadores oficiais referentes à Universidade Federal de Sergipe, definidos pelo TCU e Ministério da Educação, a fim de expor didaticamente como esse modelo pode ser implantado com o apoio de planilhas eletrônicas. Os resultados obtidos evidenciam a aplicabilidade desse modelo e reforçam a importância de que decisões gerenciais estejam sustentadas em metodologias confiáveis, capazes de considerar as preferências do decisor diante das metas institucionais, bem como o complexo ambiente acadêmico das universidades públicas brasileiras.

Palavras-chave: análise multicritério; tomada de decisão; educação superior; desempenho acadêmico; gestão pública.
\end{abstract}

\section{USE OF MULTICRITERY ANALYSIS FOR DIVISION OF FINANCIAL RESOURCES IN PUBLIC UNIVERSITIES}

\begin{abstract}
This article proposes a model based on multicriteria analysis to support decision making Prometheé - to assist in the decentralization of funding resources in public universities. For this, secondary data and official indicators referring to the Federal University of Sergipe, defined by TCU and the Ministry of Education, were used in order to expose didactically how this model can be implemented with the support of electronic spreadsheets. The results obtained show the applicability of this model and reinforce the importance of managerial decisions being supported by reliable methodologies, capable of considering the preferences of the decision maker in the light of institutional goals, as well as the complex academic environment of Brazilian public universities.

Key-words: multicriteria analysis; decision making; college education; academic achievement; public management.
\end{abstract}

\footnotetext{
1 Universidade Federal de Sergipe, Brasil. E-mail: kleber.ufs@hotmail.com.

2 Universidade Federal de Sergipe, Brasil. E-mail: thais.ettinger@academico.ufs.br.

3 Universidade Federal de Sergipe, Brasil. E-mail: zambanini@uol.com.br.

${ }^{4}$ Universidade Federal de Sergipe, Brasil. E-mail: j.jacintho50@gmail.com. 


\section{Introdução}

1 aquisição de produtos e serviços numa universidade pública é geralmente centralizada ao setor de compras que, de posse das demandas, conduz um - burocrático processo de aquisição. Guiado por uma rígida legislação esse processo depende do sucesso do certame e pode se prolongar por meses sem que 0 simples pedido seja atendido. As insatisfações surgem e possuem origem em fatores que vão desde atrasos até inconformidade com o que foi solicitado. Isto alimenta parte do que se convencionou chamar de ineficiência do serviço público.

A economicidade do processo centralizado é de certa forma bem justificada, mas se torna indefensável na medida em que possa comprometer o funcionamento das unidades ou mesmo prejudique atividades de a sala de aula, laboratórios de pesquisa, ambulatórios e consultórios.

A superação de parte dificuldades ocorreria então quando as unidades ganhassem maior autonomia para executar uma fração do orçamento de custeio para adquirir bens e serviços de uso rotineiro. Como aponta Diniz et al (2016) a descentralização tende a aumentar a agilidade nas atividades setoriais, gerando eficiência e eficácia de cada setor tanto na identificação de demandas como também usar de forma mais efetiva o conhecimento para aquisições específicas segundo decas para determinado local sejam mais fáceis de serem identificadas por seus gestores, uma vez que lidam diretamente com as demandas.

Essa autonomia requer, por sua vez, a adoção de um método eficiente de repartição dos recursos, que considere minimamente as especificidades de cada unidade, seu desempenho e, ao mesmo tempo, seja capaz de incorporar o conjunto de preferências do decisor. Esta última característica é desejável à gestão pública porque permite ordenar as unidades segundo um conjunto de preferências definidas na estratégia de desenvolvimento institucional.

No atual debate sobre a eficiência no ensino público brasileiro ${ }^{5}$ dois aspectos assumem posição de destaque: o binômio sustentabilidade-eficiência na área financeira e seu reflexo no desempenho acadêmico. Embora sejam distintos em suas atividades, o orçamentário e o acadêmico estão intimamente ligados na medida em que o aporte financeiro garante o funcionamento das unidades administrativas de ensino: cursos, departamentos e centros. Por outro lado a participação de cada universidade pública na matriz de orçamento de custeio e capital - OCC - é função do desempenho acadêmico, ou seja, da taxa de sucesso na graduação.

A despeito das delimitações legais e dificuldades operacionais na gestão financeira descentralizada, entende-se que a relativa autonomia dessas unidades pode aumentar a eficiência no atendimento de certas demandas. As questões que se impõem são: como dividir tais recursos levando em consideração o desempenho acadêmico e as especificidades de cada unidade administrativa de ensino? Como realizar essa divisão de recursos tendo em vista as metas institucionais de desempenho acadêmico? Como incorporar as preferências do decisor?

5 Uma interessante proposta de utilização da análise multicritério na gestão pública é feita por Jannuzzi et al (2009). 


\section{O método Promethée e as funções de preferência}

No conjunto de modelos aditivos lineares o método Promethée - Preference organization method for enrichment evaluation - apresenta-se como uma evolução aos procedimentos hierárquicos de auxílio à tomada de decisão. Esse método ordena alternativas segundo critérios de julgamento definidos e ponderados pela preferência do decisor. Sua aplicação depende, portanto, de uma matriz onde figurem as ' $m$ ' alternativas, os ' $n$ ' critérios e os 'w' pesos de cada critério (Gomes et al, 2004).

Outro aspecto diferencial do método Promethée, comparativamente àqueles baseados na comparação par a par, é que este método hierárquico consiste na possibilidade de associar funções contínuas aos cálculos de preferência. Tal vantagem consiste basicamente na possibilidade de se atribuir valores de preferências que sejam proporcionais à superação de um critério relativamente a outro. Em outras palavras, enquanto as funções discretas, que atribuem valor 1 às alternativas que apresentam superação em determinado critério e 0 àquelas alternativas que foram superadas, as funções contínuas atribuem valores entre 0 e 1 a essas superações de forma que pequenas diferenças entre os critérios sejam representadas por menores diferenças.

A utilização do método Promethée presume a definição do modelo de preferência. De acordo com Gomes et al (2002) o critério gaussiano apresenta algumas vantagens. A primeira delas consiste em dispensar a definição dos limiares de indiferença e de preferência estrita, aspecto que elimina os possíveis erros de resultado e interpretação decorrentes do nível de subjetividade na definição desses limiares. O segundo diferencial deste critério é o próprio padrão da função de preferência, assumindo o formato de ' $S$ ', similar à função logística, inclusive com um ponto de saturação: máximo. O procedimento envolvido também merece destaque porque requer apenas que seja calculada a variância de cada critério.

A função de preferência relativa é dada por:

$$
P_{j}\left(\delta_{i k}\right)=1-e^{\left(\frac{-\delta_{l k}^{2}}{\pi \sigma^{2}}\right)}
$$

Em que

$\delta_{\mathrm{ik}}=\left|\mathrm{u}_{\mathrm{i}}\left(\mathrm{x}_{\mathrm{i}}\right)-\mathrm{u}_{\mathrm{j}}\left(\mathrm{x}_{\mathrm{k}}\right)\right|$ é a função de superação e $\sigma^{2}$ é a variância de cada critério (indicador).

\section{Estruturação do método Promethée:}

Para melhor entendimento serão apresentados os passos para estruturação do método Promethée:

i) Estabelecendo os pesos $\left(w_{i}\right)$ : a utilização do Promethée requer que o decisor possa atribuir a cada um dos critérios os respectivos pesos. Essas ponderações podem ser valores entre 0 e 10 ou 0 a 100 ou algum outro critério. No caso desta proposta, para essa ponderação, sugere-se ferramentas de apoio às tomadas de decisão, como brainstorming ou brainwriting (PMI, 2017) em conjunto com alguma forma para reduzir a subjetividade deste tipo de ponderação, como a criação de um quadro de importância em cada um dos pilares da universidade. 
Quadro 1 -

Reduzindo a subjetividade.

\begin{tabular}{|l|l|l|l|l|l|}
\hline \multirow{2}{*}{\multicolumn{1}{|c|}{ Dimensão }} & \multicolumn{3}{c|}{ Grau de importância (pesos) } \\
\cline { 2 - 6 } & $\begin{array}{c}\text { Muito } \\
\text { baixo } \\
(0,05)\end{array}$ & $\begin{array}{c}\text { Baixo } \\
(0,10)\end{array}$ & $\begin{array}{c}\text { Moderado } \\
(0,20)\end{array}$ & $\begin{array}{c}\text { Alto } \\
(0,40)\end{array}$ & $\begin{array}{c}\text { Muito } \\
\text { alto } \\
(0,80)\end{array}$ \\
\hline Aluno equivalente na graduação (2019) & & & & & \\
\hline Taxa de sucesso na graduação (ing/dip)\% & & & & & \\
\hline Prop de matrículas \% & & & & & \\
\hline Concorrência & & & & & \\
\hline Índice de qualificação docente & & & & & \\
\hline Alun equivalente/professor & & & & & \\
\hline Trancamento/mattriculado & & & & & \\
\hline Proporção de cursos com Enade >=3 & & & & & \\
\hline Evasão/matriculados & & & & & \\
\hline Existência do curso & & & & & \\
\hline
\end{tabular}

Fonte: Elaborado pelos autores, baseados no PMI (2017)

Para utilização desse quadro cada um dos fatores deve ser inserido em um grau de importância - coluna - em cada um dos pilares universitários - linha. A soma dos três seria a ponderação desse fator.

ii) Cálculo das diferenças - desempenho - entre cada par de alternativas $\left(\delta_{\mathrm{ik}}\right)$ : Nesta etapa do modelo o decisor deve compreender que algumas variáveis utilizadas como critérios podem representar grandezas cujas direções interpretativas sejam opostas. Desta forma, as variáveis que denotam condições desejáveis à medida que assumem valores menores serão denominadas como de 'função de mínimo'; enquanto para as variáveis que denotam condições desejáveis à medida que assumem valores maiores serão denominadas como de 'função de máximo'. O estabelecimento adequado das funções de máximo ou de mínimo das alternativas é essencial para situar como se apresenta cada par de alternativa segundo determinado critério. Assim, essas diferenças ( $d_{i k}$ ) são calculadas estritamente nos casos em que exista a função de superação xiSxk, ou seja, que o desempenho da alternativa $x_{i}$ supere a alternativa $x_{i}$. Essa diferença é obtida por:

$$
\delta_{\mathrm{ik}}=\left|\mathrm{u}_{\mathrm{i}}\left(\mathrm{x}_{\mathrm{i}}\right)-\mathrm{u}_{\mathrm{j}}\left(\mathrm{x}_{\mathrm{k}}\right)\right|
$$

Observe que os cálculos dessas diferenças implicam que só existe um número se, de fato, a superação existir; caso contrário a célula correspondente fica fazia.

iii) Cálculo da função de preferência relativa: diferentemente do método hierárquico, permite que as preferências do decisor sejam modeladas por algumas funções. Este aspecto confere ao método de tomada de decisão a possibilidade de definir o poder discriminatório do critério. Uma vez estabelecido qual o critério, que no caso em estudo será o gaussiano, tem-se que a função de preferência relativa será obtida da função (1). 


\section{Cálculo do índice de preferência relativa}

Os valores das superações relativas na verdade são, na verdade, modelagens $P_{j}\left(\delta_{i k}\right)$ das superações entre critérios $\delta_{\mathrm{ik}}=\left|\mathrm{u}_{\mathrm{i}}\left(\mathrm{x}_{\mathrm{i}}\right)-\mathrm{u}_{\mathrm{j}}\left(\mathrm{x}_{\mathrm{k}}\right)\right|$, resultantes de cada par de alternativas. Interessa agora saber a intensidade dessa superação quando são considerados os pesos atribuídos pelo decisor a cada critério (Gomes et al, 2004).

$O$ índice de preferência $\left(\mathrm{s}_{\mathrm{ik}}\right)$ da alternativa $\mathrm{x}_{\mathrm{i}}$ em relação a alternativa $\mathrm{x}_{\mathrm{k}}$ é dado por:

$$
s_{\mathrm{ik}}=\frac{\Sigma_{\mathrm{j}} \mathrm{w}_{\mathrm{j}} \mathrm{P}\left(\delta_{\mathrm{ik}}\right)}{\Sigma_{\mathrm{j}} \mathrm{w}_{\mathrm{j}}}
$$

Cálculo dos índices de superação relativa - ${ }_{x i} S_{x k:}$ os valores assumidos pelos índices de preferência, resultantes da soma de cada linha ou coluna da matriz de índices de preferência permitem também a função de estabelecer as relações de comparação, conforme a seguir:

i) $x_{i}$ supera $x_{k}$ se:

$\varphi_{\mathrm{i}}^{+}>\varphi_{\mathrm{k}}^{+}$e $\varphi_{\mathrm{i}}^{-}<\varphi_{\mathrm{k}}^{-}$ou

$\varphi_{\mathrm{i}}^{+}>\varphi_{\mathrm{k}}^{+}$e $\varphi_{\mathrm{i}}^{-}=\varphi_{\mathrm{k}}^{-}$ou

$\varphi_{\mathrm{i}}^{+}=\varphi_{\mathrm{k}}^{+} \mathrm{e} \varphi_{\mathrm{i}}^{-}<\varphi_{\mathrm{k}}^{-}$

ii) $x_{i}$ é indiferente de $x_{k}$ se:

$\varphi_{\mathrm{i}}^{+}=\varphi_{\mathrm{k}}^{+}$e $\varphi_{\mathrm{i}}^{-}=\varphi_{\mathrm{k}}^{-}$ou

iii) $x_{i}$ é incomparável a $x_{k}$ se:

$\varphi_{\mathrm{i}}^{+}>\varphi_{\mathrm{k}}^{+}$e $\varphi_{\mathrm{i}}^{-}>\varphi_{\mathrm{k}}^{-}$ou

$\varphi_{\mathrm{i}}^{+}<\varphi_{\mathrm{k}}^{+}$e $\varphi_{\mathrm{i}}^{-}<\varphi_{\mathrm{k}}^{-}$

\section{Indicadores de desempenho acadêmico como critérios}

Os indicadores de desempenho acadêmico que fundamentam este artigo possuem metodologia de construção amplamente conhecida e são periodicamente publicados. Servem de exemplo os indicadores 'Aluno equivalente' e 'Taxa de sucesso', definidos pelo Tribunal de Contas da União, bem como os componentes do Exame Nacional de Desempenho dos Estudantes - Enade, em ambos os casos produzidos anualmente.

Cálculo do aluno equivalente da graduação - AEQg: o número de alunos equivalentes é essencialmente a conversão do número de alunos ingressantes e concluintes em uma métrica que considere as especificidades de cada curso, como tempo de duração, taxa de retenção, localização - se na sede ou fora - e o turno. A construção do indicador é definida pelo Ministério da Educação, pela portaria normativa n. 651, de 14 de julho de 2013, servindo inclusive como parâmetro da matriz de orçamento de custeio e capital - OCC - (Brasil, 2002), conforme a seguir:

Para os cursos já consolidados, ou seja, com mais de 10 anos de funcionamento:

$$
A E Q g_{i}^{j}=\left\{\left[D i p_{i}^{j} *\left(1+R_{i}^{j}\right)\right]+\left[\frac{\left[n g_{i}^{j}-D-D i p_{i}^{j}\right.}{4}\right]\right\} * D_{i}^{j} * B T_{i}^{j} * B F S_{i}^{j} * P G_{i}^{j}
$$

Em que

AEQgi = número de alunos equivalentes na graduação "i" no ano "j";

Dipj = número de diplomados na graduação "i" no ano "j"

$\mathrm{Di}_{\mathrm{i}}^{\mathrm{i}}=$ duração média do curso de graduação "i" 
$\mathrm{Ri}^{\mathrm{j}}$ = coeficiente de retenção da graduação "i"

Ingji-d = número de ingressantes na graduação "i" no ano "j-D"

$\mathrm{BT} \mathrm{j}$ = bônus por turno na graduação "i";

$B F S i$ = bônus por curso fora da sede na graduação "i";

$P G i j=$ peso do grupo na graduação "i";

Matrij = número de alunos matriculados no ano de referência "i".

Para os cursos novos, com menos de 10 anos, o cálculo é feito a partir de:

$A E Q g_{i}^{j}($ novos $)=M a t r_{i}^{j} * B T_{i}^{j} * B F S_{I}^{J} * P G_{I}^{J}$

O coeficiente de retenção - $R$ - refere-se ao tempo estimado de permanência do aluno além da duração do curso. Esse coeficiente é utilizado para compensar em termos de alunos equivalentes os diferenciais de retenção entre cursos. Por exemplo, para os cursos das Ciências Exatas e da Terra o fator de retenção é de 0,1325; já nos cursos de Medicina o fator é aproximadamente a metade, 0,065.

O coeficiente de duração média do curso - D - também visa a compensar os diferenciais de duração, uma vez que permanência mais prolongada requer mais recursos. Assim, mantendo os exemplos acima, tem-se que a duração média de um curso das Ciências Exatas é de 4 anos, enquanto Medicina é de 6 anos.

$O$ peso do grupo - PG - é outro coeficiente que busca mitigar diferenciais quanto à manutenção dos cursos, sobretudo referente a presença de laboratórios, oficinas, etc. Assim, um curso na área de Ciências Exatas - Computação - tem peso 2, enquanto que Medicina tem peso 4,5.

Os cursos com funcionamento noturno - BT -, e aqueles que funcionam fora da sede - BFS -, também recebem bonificações. Isto se justifica pelos gastos referentes ao consumo de energia, custos de deslocamento, de comunicação, dentre outros. Assim, os coeficientes são de $15 \%$ para os cursos noturnos e $10 \%$ para os fora da sede.

De todos os parâmetros contidos nas equações 4 e 5 , apenas o número de diplomados $\left(\mathrm{N}_{\mathrm{di}}\right)$ e de ingressantes $\left(\mathrm{N}_{\mathrm{i}}\right)$ são coletados pela instituição, ou seja, representam efetivamente a atividade acadêmica de cada lfes.

Taxa de sucesso na graduação - Suci: define-se o sucesso na graduação como a porcentagem de concluintes no ano $\mathrm{x}$ em relação aos que ingressaram no ano $\mathrm{x}-\mathrm{d}$, em que d é a duração padrão do curso.

$$
S u c_{i}=\left(\frac{D i p_{i}^{j}}{\operatorname{Ing} g_{i}^{j-a}}\right) * 100
$$

Em que

Dipi $\mathrm{i}=$ número de diplomados

Ing ${ }^{j-d_{i}}=$ número de ingressantes

Proporção de matrículas - Matri: representa a participação percentual de cada unidade no total de matrículas institucionais.

$$
T x_{-} \text {matr } r_{i}^{j}=\left(\frac{\text { Matr }_{i}^{j}}{\sum_{i=1}^{T} \text { Matr }_{i}^{j}}\right) * 100
$$


Em que

Matr $\mathrm{j}_{\mathrm{i}}=$ número de matriculados na unidade "i", no ano "j";

$\sum_{i=1}^{T} M a t r_{i}^{j}=$ total de matrículas: número de matriculados na unidade "i", no ano "j".

Concorrência no processo seletivo - Conci: mede o grau de preferência pela relação entre o número de inscritos e as respectivas vagas ofertadas por determinada unidade.

$$
\operatorname{Conc}_{i}^{j}=\left(\frac{\text { Insce }_{i}^{j}}{\sum_{i=1}^{T} I_{i n s}^{j}}\right) * 100
$$

Em que

Inscijel , i = número de inscritos na "i”, no ano “j”;

$\sum_{i=1}^{T} I n s c_{i=}^{j}=$ total de matrículas - número de matriculados na unidade "i", no ano "j".

Índice de qualificação docente - IQCDj: representa a soma de docentes ponderada pela titulação em relação ao total de doutores. Esse indicador tem resultado 5 quando todos os docentes são doutores e 1 quando são todos graduados.

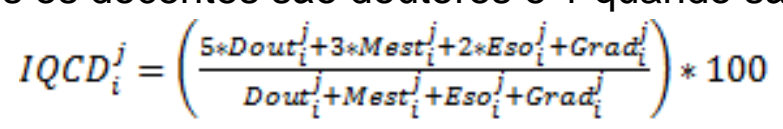

Em que

Dout $^{\dagger}, \mathrm{i}$, Mest $^{\mathrm{j}}, \mathrm{i}, \mathrm{Esp}^{\mathrm{j}}, \mathrm{i}, \mathrm{Grad}^{\mathrm{j}}, \mathrm{i}=$ número de docentes doutores, mestres, especialistas ou graduados, na unidade "i", no ano "j".

Aluno equivalente por docente - $A E_{-} D O C_{i}$ : mede a alocação de docentes por aluno equivalente. Quando esse indicador assume valores pequenos sugerem a existência de turmas pequenas.

$$
A E_{-} D O C_{i}^{j}=\left(\frac{5 * \text { Dout }_{i}^{j}+3 * \text { Mest }}{\text { Dout }_{i}^{j}+2 * E s \sigma_{i}^{j}+\operatorname{Mrad}_{i}^{j}+E s o_{i}^{j}+G r a d_{i}^{j}}\right) * 100
$$

Em que

Dout $^{\dagger}, \mathrm{i}$, Mest $^{\mathrm{j}}, \mathrm{i}, \mathrm{Esp}^{\mathrm{j}}, \mathrm{i}, \mathrm{Grad}^{\mathrm{j}}, \mathrm{i}=$ número de docentes doutores, mestres, especialistas ou graduados, na unidade "i", no ano "j".

Taxa de trancamento - Tx_Tranci: mede a proporção de alunos matriculados que realizaram trancamento total do curso ou solicitaram dispensa de matrícula.

$$
T x_{-} T r a n c_{i}^{j}=\left(\frac{\operatorname{Trand}_{i}^{j}}{\operatorname{Matr}_{i}^{j}}\right) * 100
$$

Em que

Tranc $_{\mathrm{i}}=$ número de alunos que realizaram trancamento de curso na unidade "i", no ano "j";

Matr $\mathrm{j}_{\mathrm{i}}=$ número de matriculados na unidade "i", no ano "j".

Conceito Enade - Enadeji: refere-se à proporção de cursos em cada unidade que obtiveram nota 4 ou 5 - muito bom ou excelente - no último Exame Nacional de Desempenho do Ensino Superior (Brasil, 2018) ${ }^{6}$.

\footnotetext{
6 Veja Nota técnica n. 16/2018/CGCQES/Daes.
} 
Taxa de evasão - Tx_Evasi: mede a proporção de alunos matriculados que realizaram evadiram do curso ou universidade.

$$
T x_{-} \operatorname{Evas}_{i}^{j}=\left(\frac{\text { Evas }_{i}^{j}}{\text { Matti }_{i}^{j}}\right) * 100
$$

Em que

Evas $\mathrm{j}_{\mathrm{i}}=$ número de alunos que evadiram do curso ou da universidade na unidade "i", no ano " $\mathrm{j}$ ";

Matri $\mathrm{j}_{\mathrm{i}}=$ número de matriculados na unidade "i", no ano "j".

\section{Análise dos resultados e discussão}

A Universidade Federal de Sergipe possui estrutura multicampi ${ }^{7}$, com 113 opões de cursos presenciais, com 24.520 alunos matriculados, 12 cursos na modalidade à distância sediados em 13 polos municipais com 1.520 alunos. Na pós-graduação possui 55 programas de mestrado, com 1.624 alunos, e 16 programas de doutorado que somam 754 matriculados. A essa estrutura, somam-se 1.674 docentes efetivos, 196 docentes substitutos, 2.305 servidores técnicos efetivos ou terceirizados (UFS, 2019).

O esforço gerencial necessário para movimentar essa complexa estrutura, composta por salas de aula, laboratórios, restaurante, bibliotecas e museus pode ser mais efetivo se parte da execução financeira for descentralizada ou compartilhada com os centros ou campi $^{8}$. A simulação a seguir considera a repartição de recursos de custeio entre as cinco unidades que compõem o campus Prof. José Aloísio de Campo - campus São CristóvãoSede: Centro de Ciências Exatas e Tecnologia - CCET; Centro de Educação e Ciências Humanas - CECH; Centro de Ciências Biológicas e da Saúde - CCBS; Centro de Ciências Agrárias Aplicadas - CCAA - e Centro de Ciência Sociais Aplicadas - CCSA. Estes centros congregam 16.084 alunos, o que representa $65 \%$ do total da UFS.

Para fins didáticos, este artigo utiliza informações agregadas por Centro. No entanto, essa metodologia pode ser replicada no âmbito departamental.

O plano de desenvolvimento institucional da UFS 2016-2020 estabeleceu como meta principal aumentar a taxa de sucesso de 31\% para 50\% entre 2014 e 2020 (UFS, 2016, p. 66), o que seria realizado em congruência com outras iniciativas envolvendo aprimoramento do ensino, pesquisa e extensão. O contexto em que se busca melhoria do desempenho acadêmico, em que cada unidade administrativa acadêmica pode contribuir para o alcance da meta, mostra-se oportuno também descentralizar parte dos recursos destinados ao custeio dessas unidades. Ao contrário, poder-se-ia facilmente argumentar que o não atendimento de demandas contribuiu para que a meta não fosse atingida.

A questão central é como distribuir determinado volume de recursos entre os centros do campus de São Cristóvão? A simulação a seguir mostra o grau de heterogeneidade dessas unidades, fato que não é incomum às demais universidades, mas que requer sensibilidade por parte do tomador de decisão.

\footnotetext{
7 Instalada nos municípios de São Cristóvão: campus Prof. José Aloísio de Campo/sede; Aracaju: campus João Cardoso do Nascimento Junior - Saúde/Hospital Universitário; Itabaiana: campus Prof. Alberto Carvalho; Laranjeiras: campus Laranjeiras; Lagarto: campus Prof. Antônio Garcia Filho - Saúde; e Nossa Senhora da Glória: campus do Sertão.

8 Em 2019 o total de despesas executadas pela UFS foi de $R \$ 777,05$ milhões. Conforme http://www.portaldatransparencia.gov.br/orgaos/26281?ano=2019. Acessado em 20 out. 2020.

\begin{tabular}{|l|l|l|l|l|}
\hline Regae: Rev. Gest. Aval. Educ. & Santa Maria & v. 10 & n. 19 & e48510, p. 1-16
\end{tabular}
} 
Os dados da tabela 1 mostram que em 2019 o CCET congregou 35,4\% dos alunos matriculados e $38 \%$ dos ingressantes $(x-d)^{9}$ e $38,7 \%$ dos alunos equivalentes. Esse último indicador expressa o potencial de geração de recursos via matriz OCC para a UFS. No entanto, é o CCET que também apresenta a maior participação relativa nos trancamentos e na evasão, respondendo por $35,7 \%$ e $45,6 \%$ do total do campus de São Cristóvão, respectivamente. A participação no total de docentes e doutores é também mais expressiva no CCET, ambos com 34\% desse total. $\mathrm{O} \mathrm{CECH}$ aproxima-se do CCET em alguns indicadores e noutros menos: congrega $29,5 \%$ dos matriculados, $21,3 \%$ dos alunos equivalentes e $31,2 \%$ dos trancamentos, mas $22,9 \%$ dos evadidos. No corpo docente possui $28,4 \%$ do total do campus e dos doutores. Já o CCAA é a menor unidade, com apenas $7,2 \%$ dos matriculados e $16,7 \%$ dos alunos equivalentes, possui $8,3 \%$ dos docentes e $9,2 \%$ dos doutores, $5,9 \%$ dos trancamentos e $8,4 \%$ das evasões.

Tabela 1 -

Variáveis básicas selecionadas, campus de São Cristóvão - UFS, 2019.

\begin{tabular}{c|c|c|c|c|c|c|c|c}
\hline \multirow{2}{*}{ Unidade } & \multicolumn{2}{|c|}{$\begin{array}{c}\text { Matriculados } \\
(2019)\end{array}$} & \multicolumn{2}{c|}{ Ingressante $(\mathrm{x}-\mathrm{d})$} & \multicolumn{2}{c|}{ Diplomados (2019) } & \multicolumn{2}{c}{$\begin{array}{c}\text { Aluno equivalente na } \\
\text { graduação }\end{array}$} \\
\cline { 2 - 10 } & Absoluto & $\%$ & Absoluto & $\%$ & Absoluto & $\%$ & Absoluto & $\%$ \\
\hline CCET & 5.694 & 35,40 & 1.688 & 38,72 & 366 & 25,77 & 6.864 & 37,79 \\
\hline CCBS & 1.482 & 9,21 & 373 & 8,56 & 164 & 11,55 & 2.126 & 11,71 \\
\hline CCSA & 3.015 & 18,74 & 665 & 15,26 & 289 & 20,35 & 2.259 & 12,44 \\
\hline CECH & 4.737 & 29,45 & 1.303 & 29,89 & 476 & 33,52 & 3.875 & 21,34 \\
\hline CCAA & 1.159 & 7,20 & 330 & 7,57 & 125 & 8,80 & 3.038 & 16,72 \\
\hline Total & 16.087 & 100,00 & 4.359 & 100,00 & 1.420 & 100,00 & 18.162 & 100,00 \\
\hline \multirow{2}{*}{ Unidade } & Trancamentos & \multicolumn{2}{c|}{ Evasão } & N. de professores & N. de doutores \\
\cline { 2 - 10 } & Absoluto & $\%$ & Absoluto & $\%$ & Absoluto & $\%$ & Absoluto & $\%$ \\
\hline CCET & 297 & 35,70 & 984 & 45,68 & 324 & 34,03 & 288 & 34,33 \\
\hline CCBS & 65 & 7,81 & 188 & 8,73 & 139 & 14,60 & 134 & 15,97 \\
\hline CCSA & 161 & 19,35 & 307 & 14,25 & 139 & 14,60 & 101 & 12,04 \\
\hline CECH & 260 & 31,25 & 495 & 22,98 & 271 & 28,47 & 239 & 28,49 \\
\hline CCAA & 49 & 5,89 & 180 & 8,36 & 79 & 8,30 & 77 & 9,18 \\
\hline Total & 832 & 100,00 & 2.154 & 100,00 & 952 & 100,00 & 839 & 100,00 \\
\hline
\end{tabular}

Fonte: Coordenação de Planejamento e Avaliação Acadêmica, 2020.

As variáveis acima consubstanciam a proposição dos critérios utilizados para a distribuição dos recursos por centro indicadores, conforme tabela 2.

${ }_{9} \mathrm{X}$-d indica ingresso no ano $\mathrm{x}$ menos a duração do curso.

Regae: Rev. Gest. Aval. Educ Santa Maria v. 10 
Tabela 2 -

Indicadores utilizados no modelo de alocação de recursos, campus São Cristóvão - UFS, 2019.

\begin{tabular}{l|c|c|c|c|c}
\hline Unidade & $\begin{array}{c}\text { Aluno equivalente na } \\
\text { graduação (2019) }\end{array}$ & $\begin{array}{c}\text { Taxa de } \\
\text { sucesso na } \\
\text { graduação } \\
\text { (ing/dip)\% }\end{array}$ & $\begin{array}{c}\text { Prop de } \\
\text { matrículas \% }\end{array}$ & Concorrência & $\begin{array}{c}\text { índice de } \\
\text { qualificação } \\
\text { docente }\end{array}$ \\
\hline CCET & 6.864 & 22 & 35,40 & 5 & 4,76 \\
\hline CCBS & 2.126 & 44 & 9,21 & 11 & 4,92 \\
\hline CCSA & 2.259 & 43 & 18,74 & 10 & 4,43 \\
\hline CECH & 3.875 & 37 & 29,45 & 8 & 4,75 \\
\hline CCAA & 3.038 & 38 & 7,20 & 8 & 4,95 \\
\hline Unidade & Aluno & Prop. & $\begin{array}{c}\text { Prop. } \\
\text { Diplomados ( } \\
\text { dipl/matric) }\end{array}$ & Prop evasão & Exitência curso \\
\hline CCET & 21,18 & 5,22 & 6,43 & 17,28 & 1 \\
\hline CCBS & 15,30 & 4,39 & 11,07 & 12,69 & 1 \\
\hline CCSA & 16,25 & 5,34 & 9,59 & 10,18 & 1 \\
\hline CECH & 14,30 & 5,49 & 10,05 & 10,45 & 1 \\
\hline CCAA & 38,45 & 4,23 & 10,79 & 15,53 & 0 \\
\hline
\end{tabular}

Fonte: Coordenação de Planejamento e Avaliação Acadêmica, 2020.

Para efeito de simulação admita-se que o valor a ser descentralizado seja de $R \$$ 1.000.000,00 e que os decisores avaliem que os critérios devam seguir o sistema de pesos conforme tabela 3 .

Tabela 3 -

Pesos para os critérios.

\begin{tabular}{|c|c|c|}
\hline \multirow[t]{2}{*}{ Indicadores/critérios } & $\begin{array}{l}\text { Para o seu objetivo, qual o } \\
\text { peso do critério abaixo? }\end{array}$ & \multirow[t]{2}{*}{ Peso relativo } \\
\hline & Peso absoluto (0 a 100) & \\
\hline Aluno equivalente na graduação (2019) & 50 & 18,5 \\
\hline $\begin{array}{l}\text { Taxa de sucesso na graduação } \\
\text { (ing/dip)\% }\end{array}$ & 50 & 18,5 \\
\hline Prop de matrículas \% & 50 & 18,5 \\
\hline Concorrência & 30 & 11,1 \\
\hline Índice de qualificação docente & 10 & 3,7 \\
\hline Alun equivalente/professor & 10 & 3,7 \\
\hline Trancamento/mattriculado & 30 & 11,1 \\
\hline Proporção de cursos com Enade $>=3$ & 1 & 0,4 \\
\hline Evasão/matriculados & 30 & 11,1 \\
\hline Existência do curso & 10 & 3,7 \\
\hline Total & 271 & 100,0 \\
\hline
\end{tabular}

Fonte: autores. 
Definidos os pesos, o passo inicial consiste em definir se cada critério segue função de máximo - quanto maior, melhor - ou de mínimo: quanto menor, melhor. Em seguida calcula-se para cada par as respectivas diferenças absolutas, considerando-se ainda os pesos atribuídos previamente.

Tabela 4 -

Cálculo das diferenças entre cada critério, segundo função de mínimo e máximo.

\begin{tabular}{|c|c|c|c|c|c|c|c|c|c|c|c|}
\hline & & & & & & Critérios & & & & & \\
\hline Alter & ivas & $\begin{array}{c}\text { Aluno } \\
\text { equivalent } \\
\text { e na } \\
\text { graduação }\end{array}$ & $\begin{array}{c}\text { Taxa de } \\
\text { sucesso } \\
\text { na } \\
\text { graduaçã } \\
\text { o } \\
\text { (ing/dip) } \\
\% \\
\end{array}$ & $\begin{array}{l}\text { Prop de } \\
\text { matrícul } \\
\text { as \% }\end{array}$ & $\begin{array}{c}\text { Conc } \\
\text { orrên } \\
\text { cia }\end{array}$ & $\begin{array}{c}\text { índice de } \\
\text { qualificaç } \\
\text { ão } \\
\text { docente }\end{array}$ & $\begin{array}{l}\text { Alun } \\
\text { equival } \\
\text { ente/pro } \\
\text { fessor }\end{array}$ & $\begin{array}{l}\text { Prop. } \\
\text { Tranca } \\
\text { mento }\end{array}$ & $\begin{array}{c}\text { Prop. } \\
\text { Diplom } \\
\text { ados( } \\
\text { dipl } / \mathrm{m} \\
\text { atric) }\end{array}$ & $\begin{array}{c}\text { Prop } \\
\text { evasã } \\
0\end{array}$ & $\begin{array}{c}\text { exite } \\
\text { ncia } \\
\text { curso }\end{array}$ \\
\hline Fur & & 1 & 1 & 1 & 1 & 1 & 1 & 0 & 1 & 0 & 0 \\
\hline CCET & CCBS & 4738 & & 26 & & & 6 & & & & \\
\hline CCET & CCSA & 4604 & & 17 & & 0 & 5 & 0 & & & \\
\hline CCET & $\mathrm{CECH}$ & 2989 & & 6 & & 0 & 7 & 0 & & & \\
\hline CCET & CCAA & 3826 & & 28 & & & & & & & \\
\hline CCBS & CCET & & 22 & & 6 & 0 & & 1 & 5 & 5 & \\
\hline CCBS & CCSA & & 1 & & 1 & 0 & & 1 & 1 & & \\
\hline CCBS & $\mathrm{CECH}$ & & 7 & & 3 & 0 & 1 & 1 & 1 & & \\
\hline CCBS & CCAA & & 6 & 2 & 3 & & & & 0 & 3 & \\
\hline CCSA & CCET & & 22 & & 5 & & & & 3 & 7 & \\
\hline CCSA & CCBS & 133 & & 10 & & & 1 & & & 3 & \\
\hline CCSA & $\mathrm{CECH}$ & & 7 & & 2 & & 2 & 0 & & 0 & \\
\hline CCSA & CCAA & & 6 & 12 & 2 & & & & & 5 & \\
\hline $\mathrm{CECH}$ & CCET & & 15 & & 3 & & & & 4 & 7 & \\
\hline $\mathrm{CECH}$ & CCBS & 1749 & & 20 & & & & & & 2 & \\
\hline $\mathrm{CECH}$ & CCSA & 1616 & & 11 & & 0 & & & 0 & & \\
\hline $\mathrm{CECH}$ & CCAA & 838 & & 22 & 0 & & & & & 5 & \\
\hline CCAA & CCET & & 16 & & 3 & 0 & 17 & 1 & 4 & 2 & 1 \\
\hline CCAA & CCBS & 911 & & & & 0 & 23 & 0 & & & 1 \\
\hline CCAA & CCSA & 778 & & & & 1 & 22 & 1 & 1 & & 1 \\
\hline CCAA & $\mathrm{CECH}$ & & 1 & & & 0 & 24 & 1 & 1 & & 1 \\
\hline Funcão d & ínimo (c & anto menor, & nelhor) & 0 & & & & & & & \\
\hline Funcão d & náximo ( & Ianto maior, & nelhor) & 1 & & & & & & & \\
\hline Fonte: El & rado pel & autores a p & artir dos inc & icadores & $J \Gamma 5,2$ & & & & & & \\
\hline
\end{tabular}

Os resultados acima são ajustados à função de Gaus, equação 1, de forma a indicar para cada par suas respectivas funções de preferência relativas, conforme tabela 5. 
Tabela 5 -

Cálculo das funções de preferência relativa.

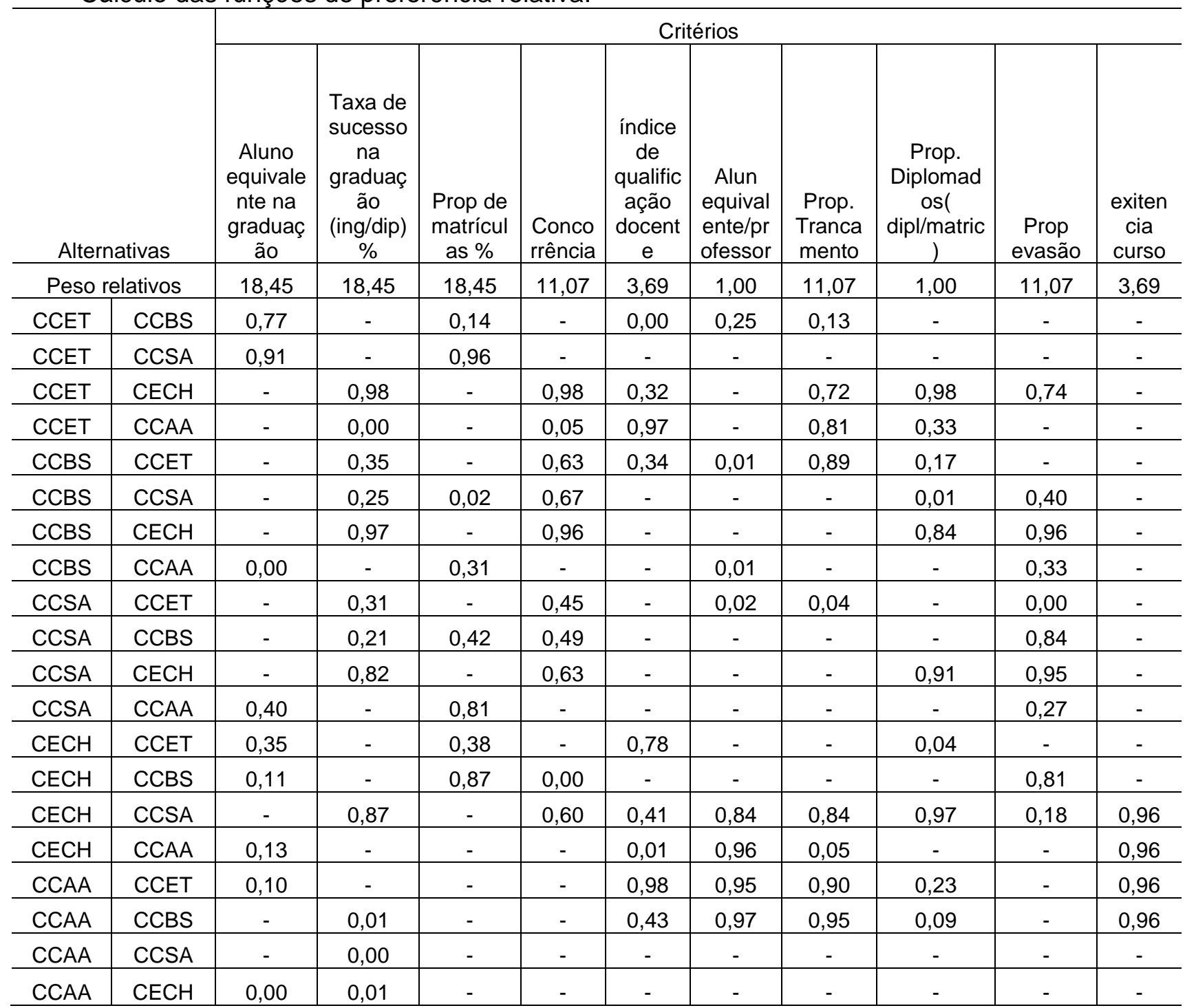

Fonte: Elaborado pelos autores a partir dos indicadores UFS, 2020.

Pelos resultados estão dispostos na tabela 6 vê-se que a participação de cada centro é proporcionalmente correspondente ao seu valor de superação positiva $\left(\square^{+}\right)$em relação à soma das superações. Desta forma, dados os desempenhos das unidades e os pesos dos critérios, tem-se que o CCET ficaria com $24,53 \%$ dos recursos ( $R \$$ $245.259,00$ ), o que daria $R \$ 43,07$ para cada aluno matriculado. Em segundo lugar o $\mathrm{CECH}$ e o CCBS receberiam cerca de $20,5 \%$ cada um (R\$204.981,64), mas considerando o número de alunos o CCBS teria $R \$ 138,25$ e o $C E C H, R \$ 43,27$. Vale ressaltar que o CCBS congrega cursos que possuem elevados custos de manutenção. $A$ menor participação seria do CCAA, com $17 \%(R \$ 170.399,03)$, o que corresponde a $R \$$ 147,02 por aluno. Deve-se ressaltar que o centro também congrega cursos que requerem elevado investimento e dispõe de Hospital Veterinário. 
Tabela 6 -

Matriz de preferências, participação relativa, valor por aluno e total a ser recebido por cada Centro.

\begin{tabular}{|c|c|c|c|c|c|c|c|c|c|c|}
\hline IJ & CCET & $\begin{array}{c}\mathrm{CCB} \\
\mathrm{S}\end{array}$ & $\begin{array}{c}\text { CCS } \\
\text { A }\end{array}$ & $\begin{array}{c}\mathrm{CEC} \\
\mathrm{H}\end{array}$ & $\begin{array}{c}\text { CCA } \\
\text { A }\end{array}$ & $\Phi^{+}$ & $\begin{array}{l}\text { Coloca- } \\
\text { ção }\end{array}$ & $\begin{array}{c}\text { Divisão } \\
\text { para cada } \\
\text { Centro } \\
(\%)\end{array}$ & $\begin{array}{l}\text { Valor por aluno } \\
\text { matriculado em } \\
\text { cada Centro }\end{array}$ & $\begin{array}{c}\text { Valor para } \\
\text { cada Centro }\end{array}$ \\
\hline CCET & & 0,36 & 0,34 & 0,18 & 0,35 & $\begin{array}{c}1,2 \\
2\end{array}$ & 1 & 24,53 & $\mathrm{R} \$ 43,07$ & $\begin{array}{c}R \$ \\
245.259,00\end{array}$ \\
\hline CCBS & 0,47 & & 0,14 & 0,25 & 0,17 & $\begin{array}{c}1,0 \\
2\end{array}$ & 3 & 20,49 & $\mathrm{R} \$ 138,25$ & $\begin{array}{c}R \$ \\
204.890,56\end{array}$ \\
\hline CCSA & 0,40 & 0,09 & & 0,11 & 0,26 & $\begin{array}{c}0,8 \\
7\end{array}$ & 4 & 17,45 & $\mathrm{R} \$ 57,87$ & $\begin{array}{c}\mathrm{R} \$ \\
174.469,77\end{array}$ \\
\hline $\begin{array}{c}\text { CEC } \\
\mathrm{H}\end{array}$ & 0,33 & 0,25 & 0,16 & & 0,27 & $\begin{array}{c}1,0 \\
2\end{array}$ & 2 & 20,50 & $\mathrm{R} \$ 43,27$ & $\begin{array}{c}\mathrm{R} \$ \\
204.981,64\end{array}$ \\
\hline CCAA & 0,41 & 0,07 & 0,20 & 0,17 & & $\begin{array}{c}0,8 \\
5\end{array}$ & 5 & 17,04 & $\mathrm{R} \$ 147,02$ & $\begin{array}{c}R \$ \\
170.399,03\end{array}$ \\
\hline$\Phi$ & 1,61 & 0,78 & 0,84 & 0,71 & 1,05 & & & 100,00 & - & $\begin{array}{c}\mathrm{R} \$ \\
1.000 .000,00\end{array}$ \\
\hline
\end{tabular}

Fonte: Elaborado pelos autores a partir dos indicadores UFS, 2020.

Uma das principais vantagens desse método consiste em incorporar um conjunto de preferências do decisor pelos pesos atribuídos aos critérios e tornar a divisão de recursos mais justa. Ocorre que a atribuição dos pesos distintos é essencialmente um procedimento subjetivo, portanto sujeito a questionamentos. Nesse sentido, o tomador de decisão pode atribuir pesos iguais a todos os critérios e assumir que não há diferenças entre os critérios utilizados.

Admita, então, que todos os critérios possuem a mesma importância e que o seu peso seja 10 , conforme a tabela 7 . A essa nova ponderação correspondem os resultados da tabela 8, que põem o CCAA em primeira posição na repartição dos recursos, com $26,22 \%$ do total $(R \$ 262.176,58)$ e valor médio por aluno de $R \$ 226,21$. Comparativamente ao CCET, que ficaria com $17,92 \%$ dos recursos ( $\$ 179.176,90)$, o que equivale a $R \$ 31,47$ por aluno. Por esse sistema de pesos o valor médio do aluno do CCAA seria 7 vezes maior que o do CCET.

Tabela 7 -

Pesos para os critérios.

\begin{tabular}{|c|c|c|}
\hline \multirow[t]{2}{*}{ Indicadores/critérios } & $\begin{array}{l}\text { Para o seu objetivo, qual o } \\
\text { peso do critério abaixo? }\end{array}$ & \multirow[t]{2}{*}{ Peso relativo } \\
\hline & Peso absoluto (0 a 100) & \\
\hline Aluno equivalente na graduação (2019) & 10 & 10,0 \\
\hline Taxa de sucesso na graduação (ing/dip)\% & 10 & 10,0 \\
\hline Prop de matrículas \% & 10 & 10,0 \\
\hline Concorrência & 10 & 10,0 \\
\hline Índice de qualificação docente & 10 & 10,0 \\
\hline Alun equivalente/professor & 10 & 10,0 \\
\hline Trancamento/mattriculado & 10 & 10,0 \\
\hline Regae: Rev. Gest. Aval. Educ. & e48510, p. & 2021 \\
\hline
\end{tabular}




\begin{tabular}{l|c|c}
\hline Proporção de cursos com Enade $>=3$ & 10 & 10,0 \\
\hline Evasão/matriculados & 10 & 10,0 \\
\hline Existência do curso & 10 & 10,0 \\
\hline Total & 100 & 100,0 \\
\hline
\end{tabular}

Fonte: autores.

Tabela 8 -

Matriz de preferências, participação relativa, valor por aluno e total a ser recebido por cada centro.

\begin{tabular}{|c|c|c|c|c|c|c|c|c|c|c|}
\hline IJ & CCET & CCBS & CCSA & $\mathrm{CECH}$ & CCAA & $\Phi^{+}$ & $\begin{array}{l}\text { Coloca- } \\
\text { ção }\end{array}$ & $\begin{array}{c}\text { Divisão } \\
\text { para cada } \\
\text { Centro } \\
(\%)\end{array}$ & $\begin{array}{c}\text { Valor por } \\
\text { aluno } \\
\text { matriculado } \\
\text { em cada } \\
\text { Centro } \\
\end{array}$ & $\begin{array}{c}\text { Valor para } \\
\text { cada Centro }\end{array}$ \\
\hline CCET & & 0,19 & 0,25 & 0,11 & 0,19 & 0,74 & 3 & 17,92 & $\mathrm{R} \$ 31,47$ & $\begin{array}{c}R \$ \\
179.176,90\end{array}$ \\
\hline CCBS & 0,38 & & 0,19 & 0,22 & 0,13 & 0,93 & 2 & 22,59 & $\mathrm{R} \$ 152,43$ & $\begin{array}{c}\mathrm{R} \$ \\
225.898,88\end{array}$ \\
\hline CCSA & 0,30 & 0,06 & & 0,08 & 0,20 & 0,64 & 5 & 15,55 & $\mathrm{R} \$ 51,58$ & $\begin{array}{c}\mathrm{R} \$ \\
155.503,30\end{array}$ \\
\hline $\mathrm{CECH}$ & 0,25 & 0,15 & 0,15 & & 0,18 & 0,73 & 4 & 17,72 & $\mathrm{R} \$ 37,42$ & $\begin{array}{c}\mathrm{R} \$ \\
177.244,39\end{array}$ \\
\hline CCAA & 0,40 & 0,12 & 0,30 & 0,25 & & 1,08 & 1 & 26,22 & $\mathrm{R} \$ 226,21$ & $\begin{array}{c}\mathrm{R} \$ \\
262.176,53\end{array}$ \\
\hline$\Phi$ & 1,33 & 0,53 & 0,89 & 0,66 & 0,70 & & & 100,00 & - & $\begin{array}{c}\mathrm{R} \$ \\
1.000 .000,00\end{array}$ \\
\hline
\end{tabular}

Fonte: Elaborado pelos autores a partir dos indicadores UFS, 2020.

Esse hiato nos valores médios chama A atenção para uma das principais vantagens da utilização desta técnica: acrescentar a preferência do decisor, além de tornar a repartição dos recursos mais democrática, visto que todos podem opinar sobre o peso dos critérios, contribui também pode tornar menos desigual a divisão de recursos entre as unidades. Veja que os hiatos valores médios obtidos com pesos diferentes - aqui adotados com o mínimo de conhecimento sobre o funcionamento dos cursos - é de 3,4 vezes ( $R \$ 147,02$ do CCAA e $R \$ 43,07$ do CCET).

A flexibilidade dessa técnica permite que a realização exaustiva de simulações seja congruente aos objetivos do desenvolvimento institucional, garantido que todos os atores sejam protagonistas nas decisões, privilegiem o desempenho e reconheçam o esforço de suas unidades sem, contudo, penalizar outras.

\section{Considerações finais}

A análise multicritério pode ser utilizada amplamente nas universidades públicas como instrumento de apoio à tomada de decisão. A utilização de indicadores oficiais, cuja metodologia é conhecida, consolidada e utilizada como instrumento de medida do desempenho acadêmico pelo TCU e MEC, tornam mais factível sua aplicação em todas as universidades públicas brasileiras.

Isto é ainda mais relevante quando se pretende fomentar que as decisões de gestão sejam baseadas na colaboração participativa da comunidade, que as desigualdades 
sejam combatidas, o esforço seja reconhecido e que as metas institucionais sejam técnicas e métodos minimamente robustos.

\section{Referências}

BRANS, Jean-Pierre, MACHARIS, Cathy, KUNSH, Pierra, SCHWANINGER, Makus. Combining multicriteria decision aid and system dynamics for the control of socioeconomic processes: an iterative real-time procedure. Eur. J. Oper. Res. North-Holland, v. 109, n. 2, 1998, p. 428-441.

BRANS, Jean-Pierre; MARESCHAL, Bertrand. Promethee methods. In: FIGUEIRA, Jose; GRECO, Salvatore; EHRGOTT, Mathias (eds.) Multiple criteria decision analysis: state of the art surveys. Boston: Springer, 2005, p. 163-196

BRASIL. Orientações para o cálculo dos indicadores de gestão: decisão TCU n. 408/2002 - plenário. Brasília: TCU, 2014.

BRASIL. Nota técnica n. 16/2018/CGCQES/Daes. Brasília: Inep, 2018.

DINIZ, Josedilton Alves et al. Impactos da descentralização na economicidade de compras governamentais. CONGRESSO CONTROLADORIA E CONTABILIDADE DA USP, 16, 2016. Anais ... São Paulo: USP, 2016. Disponível em https://congressousp.fipecafi.org/anais/artigos162016/252.pdf. Acesso em 14 jul. 2020.

GIL, Antônio Carlos. Métodos e técnicas de pesquisa social. São Paulo: Atlas, 2008

GOMES, Luiz Flávio Autran Monteiro; SIMÕES GOMES, Carlos Francisco; ALMEIDA, Adiel Teixeira de. Tomada de decisão gerencial: enfoque multicritério. São Paulo: Atlas, 2002.

GOMES, Luiz Flávio Autran Monteiro; ARAYA, Marcela Cecilia González; CARIGNANO, Claudia. Tomada de decisão em cenários complexos: introdução aos métodos discretos do apoio multicritério à decisão. São Paulo: Pioneira, Thomson Learning, 2004.

GUIMARÃES, José Ribeiro Soares; JANNUZZI, Paulo de Martino IDH, Indicadores sintéticos e suas aplicações em políticas públicas: uma análise crítica. Revista Brasileira. Est. Urbanos e Regionais, Salvador, v. 7, n. 1, 2005, p. 73-89.

JANNUZZI, Paulo de Martino; MIRANDA, Wilmer Lázaro; SILVA, Daniela Santos Gomes. Análise multicritério e tomada de decisão em políticas públicas: aspectos metodológicos, aplicativo operacional e aplicações. Informática Pública, Belo Horizonte, v. 11, n. 1, 2009, p. 69-87.

NEMERY DE BELLEVAUX, Philippe. On the use of multicriteria ranking methods in sorting problems. Bruxelles: Université Libre de Bruxelles, 2008. 302f. Thesis (Sciences de L'ingenieur). Faculté des Sciences Appliquée.

PMI. Um guia do conhecimento em gerenciamento de projetos. USA: Project Management Institute, 2017.

UFS. Plano de desenvolvimento institucional 2016-2020. Aracaju: UFS, 2016. Disponível em http://oficiais.ufs.br/uploads/page attach/path/1005/PDI-UFS 2016-2020 1 -min.pdf. Acesso em 5 jun. 2020.

UFS. Anuário estatístico 2016-2018. Aracaju: UFS, 2019. Disponível em http://indicadores.ufs.br/uploads/page attach/path/7127/Anu rio Estat stico da UFS 20 16-2017-2018 final.pdf. Acesso em 5 jun. 2020. 
Kleber Fernandes de Oliveira é professor no Departamento de Estatística e Ciências Atuariais da Universidade Federal de Sergipe.

Orcid: https://orcid.org/0000-0001-6568-6240.

Endereço: Universidade Federal de Sergipe - 49000-100 - campus São Cristóvão São Cristóvão - SE - Brasil.

E-mail: kleber.ufs@hotmail.com.

Thais Ettinger Oliveira Salgado é professor no Departamento de Administração da Universidade Federal de Sergipe.

Orcid: https://orcid.org/0000-0001-7559-2790.

Endereço: Universidade Federal de Sergipe - 49000-100 - campus São Cristóvão São Cristóvão - SE - Brasil.

E-mail: thais.ettinger@academico.ufs.br.

Marcos Eduardo Zambanini é professor no Departamento de Administração da Universidade Federal de Sergipe.

Orcid: https://orcid.org/0000-0002-0220-1514.

Endereço: Universidade Federal de Sergipe - 49000-100 - campus São Cristóvão São Cristóvão - SE - Brasil.

E-mail: zambanini@uol.com.br.

Jussara Maria Moreno Jacintho é professora na Universidade Federal de Sergipe.

Orcid: https://orcid.org/0000-0002-4717-1208.

Endereço: Universidade Federal de Sergipe - 49000-100 - campus São Cristóvão São Cristóvão - SE - Brasil.

E-mail: j.jacintho50@gmail.com.

Recebido em 30 de julho de 2020.

Aceito em 26 de novembro de 2020.

cc) (i) (s) $\odot$

BY NC ND 